\title{
Ultrasonication-Induced Synthesis and Antimicrobial Evaluation of Some Multifluorinated Pyrazolone Derivatives
}

\author{
Anil Gadhave, ${ }^{1}$ Shashikant Kuchekar, ${ }^{1}$ and Bhausaheb Karale ${ }^{2}$ \\ ${ }^{1}$ P. G. Department of Chemistry and Research Centre, Padmashri Vikhe Patil College, Pravaranagar, Ahmednagar 413713, India \\ ${ }^{2}$ Department of Chemistry, Radhabai Kale Mahila Mahavidyalaya, Ahmednagar 414001, India
}

Correspondence should be addressed to Bhausaheb Karale; bkkarale@yahoo.com

Received 6 June 2012; Revised 6 August 2012; Accepted 28 August 2012

Academic Editor: Qing Li

Copyright (c) 2013 Anil Gadhave et al. This is an open access article distributed under the Creative Commons Attribution License, which permits unrestricted use, distribution, and reproduction in any medium, provided the original work is properly cited.

\begin{abstract}
A series of novel fluorine containing pyrazole-pyrazolone $(\mathbf{4 a}-\mathbf{j})$ and chromone-pyrazolone (5a-i) was synthesized from multifluorinated pyrazolone by the Knoevenagel condensation reaction. All compounds were synthesized by conventional heating as well as ultrasound irradiation technique. It was found that ultrasonication method was more efficient than conventional heating method. The newly synthesized compounds were subjected for in vitro antimicrobial screening against four bacterial pathogens, namely, Staphylococcus aureus, Streptococcus pyogenes, Escherichia coli, and Pseudomonas aeruginosa and three fungal pathogens Candida albicans, Aspergillus niger, and Aspergillus clavatus, using broth microdilution (MIC) method (CLSI guidelines). Among them, some compounds exhibited promising antibacterial activity against the tested strains. All synthesized compounds were characterized by IR, ${ }^{1} \mathrm{H}-\mathrm{NMR}$, mass, and elemental analysis.
\end{abstract}

\section{Introduction}

An alarming increment in pathogenic resistance to existing drugs is a serious problem with antimicrobial therapy, and therefore it is very important to search for new class of antimicrobial agents [1]. The introduction of fluorine in organic molecules may lead to significant influence on the biological and physical properties of compounds due to increase of membrane permeability, hydrophobic binding, and stability against metabolic oxidation [2]. As a consequence of key role of fluorine in medicinal chemistry, the interest to incorporate fluorine to prepare biologically active compounds is increased during the past decades. The compounds containing fluorine or trifluoromethyl substituents such as fluoxetine and Prozac are well-known antidepressant drugs (Figure 1). Fluoxetine as well as the related antidepressants functions as selective serotonin reuptake inhibitors (SSRIs).

Pyrazolone is a key heterocyclic moiety present in numerous organic compounds because they possess antifungal [3], antibacterial $[4,5]$, antimycobacterial $[6,7]$, antiinflammatory $[8,9]$, antitumor [10], gastric secretion stimulatory [11], antidepressant [12], and antifilarial [13] activities. They inhibit the production of TNF- $\alpha$ and decrease levels of proinflammatory cytokines and thereby reduce inflammation and prevent further tissue destruction in disease as rheumatoid arthritis, osteoarthritis, and crohns' disease [1416]. Pyrazolone-containing drugs such as phenazone, propyphenazone, ampyrone, and metamizole are useful antipyretic and analgesic drugs [17], while edaravone (MCI-186) has been used for treating brain $[18,19]$ and myocardial ischemia [20] (Figure 1).

Pyrazole derivatives are known to possess wide spectrum of biological activities such as antibacterial [21-23], antifungal [24, 25], antidiabetic [26], herbicidal [27, 28], and antianxiety [29], and it is present as an active pharmacophore in celecoxib which is a COX-2 inhibitor [30] and sildenafil citrate as cGMP-specific phosphodiesterase type- 5 inhibitors [31]. The chromone is an important oxygen containing heterocycle present in numerous naturally occurring compounds exhibiting interesting biological activities such as antiviral [32], anticancer [33], anti-inflammatory [34, 35], and antioxidant $[36,37]$ activities.

Knoevenagel condensation reaction [38] is an important tool for construction of new carbon-carbon bonds. It is used for synthesis of different pharmaceutical products 


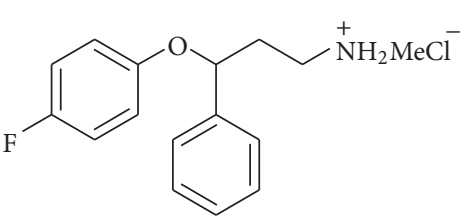

Fluoxetine hydrochloride antidepressant<smiles>CNCCC(Oc1ccc(C(F)(F)F)cc1)c1ccccc1</smiles>

Prozac antidepressant

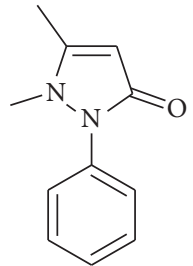

Phenazone

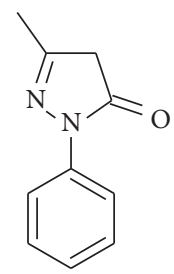

Edaravone

FIGURE 1

$[39,40]$ by using acidic and basic condition. Ultrasonication has increasingly been used for organic synthesis in the last three decades. It has been demonstrated as an alternative energy source for organic reactions ordinarily accomplished by heating. A large number of organic reactions have been carried out in higher yields, shorter reaction time, and milder conditions under ultrasound [41-44].

In view of broad spectrum of biological activities associated with pyrazolone, pyrazole, chromone nucleus, high potential of fluorine-containing compounds, and advantageous use of ultrasonication technique and in continuation to our ongoing efforts for search of biologically important heterocycles [45-47], it was envisaged to construct a system which combines all these pharmacophores in a single molecular framework to explore the additive effects towards their biological activities. Herein we wish to report green synthesis and antimicrobial evaluation of some multifluorinated pyrazolone derivatives containing pyrazole and chromone nucleus. The general route for synthesis of multifluorinated pyrazolone derivatives is outlined in Scheme 1.

\section{Experimental}

2.1. Chemistry. Melting points were taken in open capillaries and are uncorrected. The IR spectra were recorded in $\mathrm{KBr}$ on a Shimadzu FTIR 8400 spectrophotometer, and only characteristic peaks are reported in $\mathrm{cm}^{-1}$. The ${ }^{1} \mathrm{H}-\mathrm{NMR}$ spectra were recorded in DMSO- $d_{6}$ on a Bruker Avance spectrometer using TMS as an internal standard at $400 \mathrm{MHz}$, and chemical shifts are reported in parts per million ( $\mathrm{ppm}$ ). Mass spectra were scanned on a Finnigan mass spectrometer. Elemental analysis was performed on a Perkin-Elmer analyzer. Thinlayer chromatography (TLC, on aluminium plates coated with silica gel 60F254, $0.25 \mathrm{~mm}$ thickness, Merck) was used for monitoring the progress of reactions, purity, and homogeneity of the synthesized compounds. UV radiation and iodine were used as the visualizing agents. Experiment under ultrasound irradiation was carried out in ultrasonic cleaner model EN-20U-S manufactured by Enertech Electronics Pvt. Ltd., Mumbai, India, has maximum output of $100 \mathrm{~W}$ and 33 $\mathrm{KHz}$ operating frequency.

\subsection{General Procedure for Synthesis of Pyrazole-Pyrazolone (4a-j) and Chromone-Pyrazolone (5a-i)}

2.2.1. Method (A) Conventional Method. Fluorinated pyrazolone 3 (0.001 mole) and 4-formyl pyrazole or 3-formyl chromone $(0.001$ mole) were taken in $10 \mathrm{~mL}$ glacial acetic acid in $50 \mathrm{~mL}$ single neck round bottom flask equipped with condensor. The reaction mixture was heated in an oil bath at $100^{\circ} \mathrm{C}$ till completion of reaction (checked by TLC). After completion of reaction, contents were allowed to cool and then poured into crushed ice. Solid obtained was separated by filtration and purified by recrystallization from acetic acid to get pure compounds. The formation of compounds was confirmed by spectral techniques. The data of synthesized compounds is given in Tables 1 and 2 .

2.2.2. Method (B) Ultrasound Method. Fluorinated pyrazolone 3 (0.001 mole) and 4-formyl pyrazole or 3-formyl chromone $(0.001$ mole) were taken in $10 \mathrm{~mL}$ glacial acetic acid in $50 \mathrm{~mL}$ round bottom flask. Contents of flask were subjected for ultrasound irradiation for time as shown in Tables 1 and 2, till completion of reaction (checked by TLC). After completion of reaction, contents were poured into crushed ice. Solid obtained was separated by filtration and purified by recrystallization from acetic acid to get pure compounds. The formation of compounds was confirmed by $\mathrm{mp}$, mixed $\mathrm{mp}$, and spectral techniques. The physical data of synthesized compounds is given in Tables 1 and 2 .

(4Z)-4-[(1,3-Diphenyl-1H-pyrazol-4-yl)methylene]-5-propyl2-(2,3,5,6-tetrafluorophenyl)-2,4-dihydro-3H-pyrazol-3-one

(4a). Yellow solid; IR $\left(\nu_{\max }, \mathrm{cm}^{-1}\right): 1688$ (C=O lactum), 1595 $(\mathrm{C}=\mathrm{C}), 1515(\mathrm{C}=\mathrm{N}), 1121(\mathrm{Ar}-\mathrm{F}) ;{ }^{1} \mathrm{H}-\mathrm{NMR} \delta(400 \mathrm{MHz}$, DMSO- $\left.d_{6}\right): 1.01-1.04(\mathrm{t}, 3 \mathrm{H}, J=12 \mathrm{~Hz}), 1.71-1.79(\mathrm{~m}$, $2 \mathrm{H}), 2.56-2.60(\mathrm{t}, 2 \mathrm{H}, J=16 \mathrm{~Hz}), 7.13-7.18(\mathrm{~m}, 1 \mathrm{H}, \mathrm{Ar}-\mathrm{H})$, 7.35-7.39 (m, 1H, Ar-H), 7.46-7.51 (m, 2H, Ar-H), 7.54-7.60 (m, 4H, Ar-H), 7.66-7.69 (m, 2H, Ar-H), 7.87-7.90 ( $\mathrm{m}, 2 \mathrm{H}$, one proton $\mathrm{Ar}-\mathrm{H}$ \& one pyrazole proton), 10.16 (s, $1 \mathrm{H}$, olefinic proton); MS: $m / z 504\left(\mathrm{M}^{+}\right)$; Anal. Calcd for $\mathrm{C}_{28} \mathrm{H}_{20} \mathrm{~F}_{4} \mathrm{~N}_{4} \mathrm{O}$ : C, 66.66; $\mathrm{H}, 4.00 ; \mathrm{N}, 11.11$. Found: C, 66.71; H, 3.92; N, 11.04 .

(4Z)-4-\{[3-(4-Fluorophenyl)-1-phenyl-1H-pyrazol-4-yl] methylene $\}$-5-propyl-2-(2,3,5,6-tetrafluorophenyl)-2,4dihydro-3H-pyrazol-3-one (4b). Yellow solid; IR ( $v_{\max }$, $\left.\mathrm{cm}^{-1}\right)$ : 1697 (C=O lactum), $1580(\mathrm{C}=\mathrm{C}), 1521(\mathrm{C}=\mathrm{N})$, 1133 (Ar-F); ${ }^{1} \mathrm{H}-\mathrm{NMR} \delta\left(400 \mathrm{MHz}, \mathrm{DMSO}-d_{6}\right): 1.01-1.05$ $(\mathrm{t}, 3 \mathrm{H}, J=16 \mathrm{~Hz}), 1.73-1.79(\mathrm{~m}, 2 \mathrm{H}), 2.56-2.60(\mathrm{t}, 2 \mathrm{H}$, $J=16 \mathrm{~Hz}), 7.13-7.18(\mathrm{~m}, 1 \mathrm{H}, \mathrm{Ar}-\mathrm{H}), 7.25-7.29(\mathrm{~m}, 2 \mathrm{H}$, Ar-H), 7.36-7.40 (m, 1H, Ar-H), 7.47-7.52 (m, 3H, Ar-H), 7.64-7.68 (m, 2H, Ar-H), 7.86-7.89 (m, 2H, one proton Ar-H \& one pyrazole proton), 10.15 (s, 1H, olefinic proton); 
TABle 1: Physical data of synthesized pyrazole-pyrazolone (4a-j).

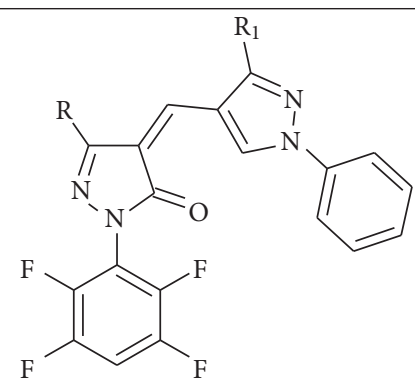

\begin{tabular}{|c|c|c|c|c|c|c|c|}
\hline \multirow{2}{*}{ Compound } & \multirow{2}{*}{$\mathrm{R}$} & \multirow{2}{*}{$\mathrm{R}_{1}$} & \multirow{2}{*}{ M.P. $\left({ }^{\circ} \mathrm{C}\right)$} & \multicolumn{2}{|c|}{ Conventional method } & \multicolumn{2}{|c|}{ Ultrasound method } \\
\hline & & & & Time (min) & Yield (\%) & Time (min) & Yield (\%) \\
\hline $4 a$ & Propyl & $\mathrm{C}_{6} \mathrm{H}_{5}$ & $151-152$ & 60 & 75 & 16 & 90 \\
\hline $4 b$ & Propyl & $4-\mathrm{FC}_{6} \mathrm{H}_{4}$ & $179-180$ & 60 & 71 & 15 & 91 \\
\hline $4 c$ & Propyl & $4-\mathrm{MeC}_{6} \mathrm{H}_{4}$ & $192-193$ & 60 & 69 & 18 & 88 \\
\hline $4 d$ & Propyl & $4-\mathrm{ClC}_{6} \mathrm{H}_{4}$ & $159-160$ & 60 & 73 & 16 & 85 \\
\hline $4 e$ & Propyl & 2-Thienyl & $149-150$ & 60 & 66 & 19 & 90 \\
\hline $4 \mathrm{f}$ & $\mathrm{CF}_{3}$ & $\mathrm{C}_{6} \mathrm{H}_{5}$ & $237-238$ & 60 & 78 & 21 & 86 \\
\hline $4 \mathrm{~g}$ & $\mathrm{CF}_{3}$ & $4-\mathrm{FC}_{6} \mathrm{H}_{4}$ & $235-236$ & 60 & 74 & 19 & 93 \\
\hline $4 h$ & $\mathrm{CF}_{3}$ & $4-\mathrm{MeC}_{6} \mathrm{H}_{4}$ & 253-254 & 60 & 71 & 18 & 89 \\
\hline $4 \mathbf{i}$ & $\mathrm{CF}_{3}$ & $4-\mathrm{ClC}_{6} \mathrm{H}_{4}$ & $216-217$ & 60 & 76 & 17 & 94 \\
\hline $4 j$ & $\mathrm{CF}_{3}$ & 2-Thienyl & $215-216$ & 60 & 69 & 16 & 90 \\
\hline
\end{tabular}

TABLE 2: Physical data of synthesized chromone-pyrazolone (5a-i).

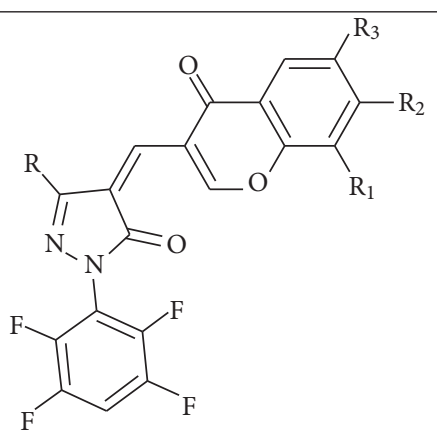

\begin{tabular}{|c|c|c|c|c|c|c|c|c|c|}
\hline \multirow{2}{*}{ Compound } & \multirow{2}{*}{$\mathrm{R}$} & \multirow{2}{*}{$\mathrm{R}_{1}$} & \multirow{2}{*}{$\mathrm{R}_{2}$} & \multirow{2}{*}{$\mathrm{R}_{3}$} & \multirow{2}{*}{ M. P. $\left({ }^{\circ} \mathrm{C}\right)$} & \multicolumn{2}{|c|}{ Conventional method } & \multicolumn{2}{|c|}{ Ultrasound method } \\
\hline & & & & & & Time (min) & Yield (\%) & Time (min) & Yield (\%) \\
\hline $5 \mathbf{a}$ & Propyl & $\mathrm{H}$ & $\mathrm{H}$ & $\mathrm{H}$ & $144-145$ & 60 & 70 & 14 & 81 \\
\hline $5 \mathbf{b}$ & Propyl & $\mathrm{H}$ & $\mathrm{H}$ & $\mathrm{Me}$ & $146-147$ & 60 & 68 & 16 & 80 \\
\hline $5 c$ & Propyl & $\mathrm{Cl}$ & $\mathrm{H}$ & $\mathrm{Cl}$ & 194-195 & 60 & 69 & 13 & 82 \\
\hline $5 d$ & Propyl & $\mathrm{H}$ & $\mathrm{H}$ & $\mathrm{Cl}$ & $166-167$ & 60 & 72 & 17 & 78 \\
\hline $5 e$ & Propyl & $\mathrm{H}$ & $\mathrm{Me}$ & $\mathrm{Cl}$ & $241-242$ & 60 & 75 & 12 & 82 \\
\hline $5 f$ & $\mathrm{CF}_{3}$ & $\mathrm{H}$ & $\mathrm{H}$ & $\mathrm{H}$ & $222-223$ & 60 & 71 & 14 & 85 \\
\hline $5 \mathrm{~g}$ & $\mathrm{CF}_{3}$ & $\mathrm{H}$ & $\mathrm{H}$ & $\mathrm{Me}$ & $238-239$ & 60 & 66 & 15 & 81 \\
\hline $5 \mathrm{~h}$ & $\mathrm{CF}_{3}$ & $\mathrm{Cl}$ & $\mathrm{H}$ & $\mathrm{Cl}$ & $212-213$ & 60 & 69 & 12 & 82 \\
\hline $5 \mathbf{i}$ & $\mathrm{CF}_{3}$ & $\mathrm{H}$ & $\mathrm{H}$ & $\mathrm{Cl}$ & $208-209$ & 60 & 71 & 16 & 84 \\
\hline
\end{tabular}


<smiles>[R]C(=O)CC(=O)OCC</smiles>

1<smiles>NNc1c(F)c(F)cc(F)c1F</smiles>

2

$120-130^{\circ} \mathrm{C}$

solvent free, 3 hours<smiles>[R]C1=NN(c2c(F)c(F)cc(F)c2F)C(=O)C1</smiles>

3

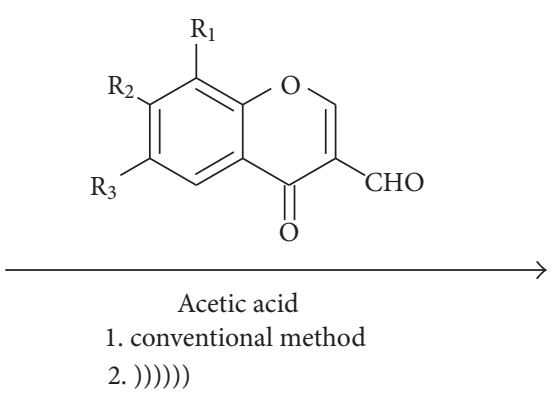

2. $))())$ )<smiles>[R]C1=NN(c2c(F)c(F)cc(F)c2F)C(=O)/C1=C\c1coc2c([R1])c([R2])c([R3])cc2c1=O</smiles><smiles>[R]c1nn(-c2ccccc2)cc1C=O</smiles><smiles>[R]C1=NN(c2c(F)c(F)cc(F)c2F)C(=O)/C1=C\c1cn(-c2ccccc2)nc1[R]</smiles>

SCHEme 1: Synthesis of multifluorinated pyrazolone containing pyrazole and chromone.

MS: $m / z 522\left(\mathrm{M}^{+}\right)$; Anal. Calcd for $\mathrm{C}_{28} \mathrm{H}_{19} \mathrm{~F}_{5} \mathrm{~N}_{4} \mathrm{O}$ : C, 64.37; H, 3.67; N, 10.72. Found: C, 64.50; H, 3.61; N, 10.65 .

(4Z)-4-\{[3-(4-Methylphenyl)-1-phenyl-1H-pyrazol-4yl]methylene -5-propyl-2-(2,3,5,6-tetrafluorophenyl)-2,4dihydro-3H-pyrazol-3-one (4c). Yellow solid; IR $\left(v_{\max }, \mathrm{cm}^{-1}\right)$ :
$1693(\mathrm{C}=\mathrm{O}$ lactum), $1597(\mathrm{C}=\mathrm{C}), 1505(\mathrm{C}=\mathrm{N}), 1114(\mathrm{Ar}-\mathrm{F})$; ${ }^{1} \mathrm{H}-\mathrm{NMR} \delta\left(400 \mathrm{MHz}, \mathrm{DMSO}-d_{6}\right): 0.99-1.03(\mathrm{t}, 3 \mathrm{H}, J=$ $16 \mathrm{~Hz}), 1.72-1.77(\mathrm{~m}, 2 \mathrm{H}), 2.46\left(\mathrm{~s}, 3 \mathrm{H}, \mathrm{Ar}-\mathrm{CH}_{3}\right), 2.56-2.58$ $(\mathrm{t}, 2 \mathrm{H}, J=8 \mathrm{~Hz}), 7.09-7.17(\mathrm{~m}, 1 \mathrm{H}, \mathrm{Ar}-\mathrm{H}), 7.35-7.38(\mathrm{~m}$, $3 \mathrm{H}, \mathrm{Ar}-\mathrm{H}), 7.44-7.48(\mathrm{~m}, 2 \mathrm{H}, \mathrm{Ar}-\mathrm{H}), 7.54-7.56(\mathrm{~d}, 2 \mathrm{H}, J=$ $8 \mathrm{~Hz}, \mathrm{Ar}-\mathrm{H}), 7.59$ (s, $1 \mathrm{H}$, pyrazole proton), 7.85-7.87 (d, $2 \mathrm{H}$, 
$J=8 \mathrm{~Hz}, \mathrm{Ar}-\mathrm{H}$ ), 10.13 (s, $1 \mathrm{H}$, olefinic proton); MS: $m / z 518$ $\left(\mathrm{M}^{+}\right)$; Anal. Calcd for $\mathrm{C}_{29} \mathrm{H}_{22} \mathrm{~F}_{4} \mathrm{~N}_{4} \mathrm{O}: \mathrm{C}, 67.18 ; \mathrm{H}, 4.28 ; \mathrm{N}$, 10.81. Found: C, $67.11 ; \mathrm{H}, 4.37 ; \mathrm{N}, 10.89$.

(4Z)-4-\{[3-(4-Chlorophenyl)-1-phenyl-1H-pyrazol-4-yl]methylene\}-5-propyl-2-(2,3,5,6-tetrafluorophenyl)-2,4dihydro-3H-pyrazol-3-one (4d). Yellow solid; IR ( $v_{\max }$, $\left.\mathrm{cm}^{-1}\right): 1681(\mathrm{C}=\mathrm{O}$ lactum $), 1583(\mathrm{C}=\mathrm{C}), 1526(\mathrm{C}=\mathrm{N})$, 1125 (Ar-F); ${ }^{1} \mathrm{H}-\mathrm{NMR} \delta\left(400 \mathrm{MHz}, \mathrm{DMSO}-d_{6}\right): 1.02-1.06$ $(\mathrm{t}, 3 \mathrm{H}, J=16 \mathrm{~Hz}), 1.74-1.79(\mathrm{~m}, 2 \mathrm{H}), 2.57-2.60(\mathrm{t}, 2 \mathrm{H}$, $J=12 \mathrm{~Hz}), 7.13-7.18(\mathrm{~m}, 1 \mathrm{H}, \mathrm{Ar}-\mathrm{H}), 7.36-7.40(\mathrm{~m}, 1 \mathrm{H}$, Ar-H), 7.47-7.52 (m, 3H, Ar-H), 7.54-7.56 (m, 2H, Ar-H), 7.62-7.63 ( $\mathrm{m}, 2 \mathrm{H}$, one proton $\mathrm{Ar}-\mathrm{H} \&$ one pyrazole proton), 7.86-7.88 (m, 2H, Ar-H), 10.16 (s, 1H, olefinic proton); MS: $m / z 538\left(\mathrm{M}^{+}\right)$; Anal. Calcd for $\mathrm{C}_{28} \mathrm{H}_{19} \mathrm{ClF}_{4} \mathrm{~N}_{4} \mathrm{O}: \mathrm{C}, 62.40 ; \mathrm{H}$, $3.55 ; \mathrm{N}, 10.40$. Found: C, 62.30; H, 3.61; N, 10.35 .

(4Z)-4-\{[1-Phenyl-3-(2-thienyl)-1H-pyrazol-4-yl]methylene $\}$ 5-propyl-2-(2,3,5,6-tetrafluorophenyl)-2,4-dihydro-3H-

pyrazol-3-one (4e). Yellow solid; IR $\left(v_{\max }, \mathrm{cm}^{-1}\right): 1688(\mathrm{C}=\mathrm{O}$ lactum), 1603 (C=C), 1509 (C=N), 1101 (Ar-F); ${ }^{1} \mathrm{H}-\mathrm{NMR}$ $\delta\left(400 \mathrm{MHz}, \mathrm{DMSO}-d_{6}\right): 1.05-1.08(\mathrm{t}, 3 \mathrm{H}, J=12 \mathrm{~Hz})$, 1.79-1.85 (m, 2H), 2.65-2.68 (t, $2 \mathrm{H}, J=12 \mathrm{~Hz}), 7.13-7.18$ (m, 1H, Ar-H), 7.24-7.26 (m, 1H, Ar-H), 7.35-7.38 (m, 1H, Ar-H), 7.45-7.51 (m, 3H, Ar-H), 7.54-7.55 (m, 1H, Ar-H), $7.83(\mathrm{~s}, 1 \mathrm{H}$, pyrazole proton), 7.86-7.88 (m, $2 \mathrm{H}, \mathrm{Ar}-\mathrm{H})$, 10.16 (s, $1 \mathrm{H}$, olefinic proton); MS: $m / z 510\left(\mathrm{M}^{+}\right)$; Anal. Calcd for $\mathrm{C}_{26} \mathrm{H}_{18} \mathrm{~F}_{4} \mathrm{~N}_{4} \mathrm{OS}$ : C, 61.17; $\mathrm{H}, 3.55 ; \mathrm{N}, 10.97$. Found: $\mathrm{C}$, $61.29 ; \mathrm{H}, 3.49 ; \mathrm{N}, 10.85$.

(4Z)-4-[(1,3-Diphenyl-1H-pyrazol-4-yl)methylene]-2-(2,3, 5,6-tetrafluorophenyl)-5-(trifluoromethyl)-2,4-dihydro-3Hpyrazol-3-one (4f). Yellow solid; IR $\left(v_{\max }, \mathrm{cm}^{-1}\right): 1701(\mathrm{C}=\mathrm{O}$ lactum), $1591(\mathrm{C}=\mathrm{C}), 1515(\mathrm{C}=\mathrm{N}), 1112(\mathrm{Ar}-\mathrm{F}) ;{ }^{1} \mathrm{H}-\mathrm{NMR}$ $\delta\left(400 \mathrm{MHz}, \mathrm{DMSO}-d_{6}\right): 7.20(\mathrm{~s}, 1 \mathrm{H}, \mathrm{Ar}-\mathrm{H}), 7.41-7.44(\mathrm{~m}$, $1 \mathrm{H}, \mathrm{Ar}-\mathrm{H}), 7.46-7.57$ (m, 5H, Ar-H), 7.76 (s, 2H, Ar-H), 7.81-7.92 (m, 2H, Ar-H), 7.99 (s, $1 \mathrm{H}$, pyrazole proton), 10.23 (s, $1 \mathrm{H}$, olefinic proton); MS: $m / z 530\left(\mathrm{M}^{+}\right)$; Anal. Calcd for $\mathrm{C}_{26} \mathrm{H}_{13} \mathrm{~F}_{7} \mathrm{~N}_{4} \mathrm{O}$ : C, 58.88; H, 2.47; N, 10.56. Found: C, $58.80 ; \mathrm{H}, 2.51 ; \mathrm{N}, 10.50$.

(4Z)-4-\{[3-(4-Fluorophenyl)-1-phenyl-1H-pyrazol-4-yl] methylene\}-2-(2,3,5,6-tetrafluorophenyl)-5-(trifluoromethyl)2,4-dihydro-3H-pyrazol-3-one (4g). Yellow solid; IR ( $v_{\max }$, $\left.\mathrm{cm}^{-1}\right): 1691(\mathrm{C}=\mathrm{O}$ lactum), $1609(\mathrm{C}=\mathrm{C}), 1522(\mathrm{C}=\mathrm{N}), 1130$ (Ar-F); ${ }^{1} \mathrm{H}-\mathrm{NMR} \delta\left(400 \mathrm{MHz}, \mathrm{DMSO}-d_{6}\right): 7.27-7.31(\mathrm{~m}$, $3 \mathrm{H}, \mathrm{Ar}-\mathrm{H}), 7.42-7.52$ (m, 3H, Ar-H), 7.66 (s, 2H, Ar-H), 7.88-7.91 ( $\mathrm{m}, 3 \mathrm{H}$, two proton Ar-H \& one pyrazole proton), 10.22 (s, $1 \mathrm{H}$, olefinic proton); MS: $m / z 548\left(\mathrm{M}^{+}\right)$; Anal. Calcd for $\mathrm{C}_{26} \mathrm{H}_{12} \mathrm{~F}_{8} \mathrm{~N}_{4} \mathrm{O}: \mathrm{C}, 56.94 ; \mathrm{H}, 2.21 ; \mathrm{N}, 10.22$. Found: C, 56.90; H, 2.30; N, 10.28 .

(4Z)-4-\{[3-(4-Methylphenyl)-1-phenyl-1H-pyrazol-4-yl] methylene\}-2-(2,3,5,6-tetrafluorophenyl)-5-(trifluoromethyl)2,4-dihydro-3H-pyrazol-3-one $(\mathbf{4} \mathbf{~ h})$. Yellow solid; IR ( $v_{\max }$, $\left.\mathrm{cm}^{-1}\right)$ : $1708(\mathrm{C}=\mathrm{O}$ lactum $), 1600(\mathrm{C}=\mathrm{C}), 1510(\mathrm{C}=\mathrm{N}), 1122$ $(\mathrm{Ar}-\mathrm{F}) ;{ }^{1} \mathrm{H}-\mathrm{NMR} \delta\left(400 \mathrm{MHz}, \mathrm{DMSO}-d_{6}\right): 2.46(\mathrm{~s}, 3 \mathrm{H}$, Ar- $\left.\mathrm{CH}_{3}\right), 7.19-7.21(\mathrm{~m}, 1 \mathrm{H}, \mathrm{Ar}-\mathrm{H}), 7.35-7.40(\mathrm{~m}, 3 \mathrm{H}$,
Ar-H), 7.47-7.50 (m, 2H, Ar-H), 7.54-7.56 (d, 2H, $J=$ $8 \mathrm{~Hz}, \mathrm{Ar}-\mathrm{H}), 7.87-7.89$ (d, $2 \mathrm{H}, J=8 \mathrm{~Hz}, \mathrm{Ar}-\mathrm{H}), 7.99$ (s, $1 \mathrm{H}$, pyrazole proton), 10.20 (s, $1 \mathrm{H}$, olefinic proton); MS: $\mathrm{m} / z 544$ $\left(\mathrm{M}^{+}\right)$; Anal. Calcd for $\mathrm{C}_{27} \mathrm{H}_{15} \mathrm{~F}_{7} \mathrm{~N}_{4} \mathrm{O}: \mathrm{C}, 59.57 ; \mathrm{H}, 2.78$; N, 10.29. Found: C, 59.65; H, 2.70; N, 10.20 .

(4Z)-4-\{[3-(4-Chlorophenyl)-1-phenyl-1H-pyrazol4-yl]methylene $\}-2-(2,3,5,6$-tetrafluorophenyl)-5(trifluoromethyl)-2,4-dihydro-3H-pyrazol-3-one (4i). Yellow solid; IR $\left(v_{\max }, \mathrm{cm}^{-1}\right)$ : 1711 (C=O lactum), $1599(\mathrm{C}=\mathrm{C}), 1501$ $(\mathrm{C}=\mathrm{N}), 1109(\mathrm{Ar}-\mathrm{F}) ;{ }^{1} \mathrm{H}-\mathrm{NMR} \delta\left(400 \mathrm{MHz}, \mathrm{DMSO}-d_{6}\right)$ : 7.26 (s, 1H, Ar-H), 7.42-7.61 (m, 7H, Ar-H), 7.87-7.91 (m, $3 \mathrm{H}$, two proton Ar-H \& one pyrazole proton), 10.23 (s, $1 \mathrm{H}$, olefinic proton); MS: $m / z 564\left(\mathrm{M}^{+}\right)$, Anal. Calcd for $\mathrm{C}_{26} \mathrm{H}_{12} \mathrm{ClF}_{7} \mathrm{~N}_{4} \mathrm{O}$ : C, 55.29; H, 2.14; N, 9.92. Found: C, 55.21; $\mathrm{H}, 2.20 ; \mathrm{N}, 9.81$.

(4Z)-4-\{[1-Phenyl-3-(2-thienyl)-1H-pyrazol-4-yl]methylene $\}$ 2-(2,3,5,6-tetrafluorophenyl)-5-(trifluoromethyl)-2,4-dihydro3H-pyrazol-3-one (4j). Yellow solid; IR $\left(v_{\max }, \mathrm{cm}^{-1}\right): 1701$ $(\mathrm{C}=\mathrm{O}$ lactum $), 1605(\mathrm{C}=\mathrm{C}), 1505(\mathrm{C}=\mathrm{N}), 1116(\mathrm{Ar}-\mathrm{F})$; ${ }^{1} \mathrm{H}-\mathrm{NMR} \delta\left(400 \mathrm{MHz}, \mathrm{DMSO}-d_{6}\right): 7.22(\mathrm{~s}, 1 \mathrm{H}, \mathrm{Ar}-\mathrm{H})$, 7.41-7.56 (m, 6H, Ar-H), 7.85-7.89 (m, 2H, Ar-H), 8.22 (s, $1 \mathrm{H}$, pyrazole proton), 10.20 (s, $1 \mathrm{H}$, olefinic proton); MS: $\mathrm{m} / \mathrm{z}$ $536\left(\mathrm{M}^{+}\right)$; Anal. Calcd for $\mathrm{C}_{24} \mathrm{H}_{11} \mathrm{~F}_{7} \mathrm{~N}_{4} \mathrm{OS}$ : C, 53.74; $\mathrm{H}, 2.07$; $\mathrm{N}, 10.44$. Found: C, $53.69 ; \mathrm{H}, 2.10 ; \mathrm{N}, 10.55$.

(4Z)-4-[(4-Oxo-4H-chromen-3-yl)methylene]-5-propyl-2(2,3,5,6-tetrafluorophenyl)-2,4-dihydro-3H-pyrazol-3-one

(5a). Yellow solid; IR $\left(v_{\max }, \mathrm{cm}^{-1}\right): 1700(\mathrm{C}=\mathrm{O}$ lactum $), 1659$ (C=O chromone), $1623(\mathrm{C}=\mathrm{C}), 1599(\mathrm{C}=\mathrm{N}), 1129(\mathrm{Ar}-\mathrm{F})$; ${ }^{1} \mathrm{H}-\mathrm{NMR} \delta\left(400 \mathrm{MHz}, \mathrm{DMSO}-d_{6}\right): 0.99-1.02(\mathrm{t}, 3 \mathrm{H}, J=$ $12 \mathrm{~Hz}), 1.69-1.75(\mathrm{~m}, 2 \mathrm{H}), 2.71-2.74(\mathrm{t}, 2 \mathrm{H}, J=12 \mathrm{~Hz})$, 7.59-7.63 (m, 1H, Ar-H), 7.79-7.81 (d, $1 \mathrm{H}, J=8 \mathrm{~Hz}, \mathrm{Ar}-\mathrm{H})$, 7.90-7.94 (m, 1H, Ar-H), 8.05 (s, 1H, Ar-H), 8.11-8.19 (m, $2 \mathrm{H}, \mathrm{Ar}-\mathrm{H}), 10.35$ (s, $1 \mathrm{H}$, olefinic proton); MS: $m / z 430\left(\mathrm{M}^{+}\right)$; Anal. Calcd for $\mathrm{C}_{22} \mathrm{H}_{14} \mathrm{~F}_{4} \mathrm{~N}_{2} \mathrm{O}_{3}$ : C, 61.40; $\mathrm{H}, 3.28 ; \mathrm{N}, 6.51$. Found: C, 61.31; H, 3.22; N, 6.61.

(4Z)-4-[(6-Methyl-4-oxo-4H-chromen-3-yl)methylene]-5propyl-2-(2,3,5,6-tetrafluorophenyl)-2,4-dihydro-3H-pyrazol3-one (5b). Yellow solid; IR $\left(v_{\max }, \mathrm{cm}^{-1}\right): 1694(\mathrm{C}=\mathrm{O}$ lactum), 1669 ( $\mathrm{C}=\mathrm{O}$ chromone), $1627(\mathrm{C}=\mathrm{C}), 1588(\mathrm{C}=\mathrm{N})$, 1140 (Ar-F); ${ }^{1} \mathrm{H}-\mathrm{NMR} \delta\left(400 \mathrm{MHz}, \mathrm{DMSO}-d_{6}\right): 0.97-1.01$ $(\mathrm{t}, 3 \mathrm{H}, J=16 \mathrm{~Hz}), 1.68-1.73(\mathrm{~m}, 2 \mathrm{H}), 2.45\left(\mathrm{~s}, 3 \mathrm{H}, \mathrm{Ar}-\mathrm{CH}_{3}\right)$, 2.69-2.72 (t, $2 \mathrm{H}, J=12 \mathrm{~Hz}), 7.66-7.72(\mathrm{~m}, 2 \mathrm{H}, \mathrm{Ar}-\mathrm{H}), 7.95$ (s, 1H, Ar-H), 8.03 (s, 1H, Ar-H), 8.07-8.11 (m, 1H, Ar-H), 10.35 (s, $1 \mathrm{H}$, olefinic proton); MS: $\mathrm{m} / z 444\left(\mathrm{M}^{+}\right)$; Anal. Calcd for $\mathrm{C}_{23} \mathrm{H}_{16} \mathrm{~F}_{4} \mathrm{~N}_{2} \mathrm{O}_{3}$ : C, 62.16; H, 3.63; N, 6.30. Found: C, $62.24 ; \mathrm{H}, 3.59 ; \mathrm{N}, 6.23$.

(4Z)-4-[(6,8-Dichloro-4-oxo-4H-chromen-3-yl)methylene]-5propyl-2-(2,3,5,6-tetrafluorophenyl)-2,4-dihydro-3H-pyrazol3-one (5c). Yellow solid; IR $\left(v_{\max }, \mathrm{cm}^{-1}\right): 1707(\mathrm{C}=\mathrm{O}$ lactum), $1659(\mathrm{C}=\mathrm{O}$ chromone $), 1624(\mathrm{C}=\mathrm{C}), 1577(\mathrm{C}=\mathrm{N})$, 1126 (Ar-F); ${ }^{1} \mathrm{H}-\mathrm{NMR} \delta\left(400 \mathrm{MHz}, \mathrm{DMSO}-d_{6}\right): 1.03-1.07$ $(\mathrm{t}, 3 \mathrm{H}, J=12 \mathrm{~Hz}), 1.77-1.82(\mathrm{~m}, 2 \mathrm{H}), 2.68-2.71(\mathrm{t}, 2 \mathrm{H}, J=$ $12 \mathrm{~Hz}), 7.10-7.16(\mathrm{~m}, 1 \mathrm{H}, \mathrm{Ar}-\mathrm{H}), 7.77(\mathrm{~s}, 1 \mathrm{H}, \mathrm{Ar}-\mathrm{H}), 7.99$ (s, 
$1 \mathrm{H}, \mathrm{Ar}-\mathrm{H}), 8.13$ (s, 1H, Ar-H), 10.66 (s, 1H, olefinic proton); MS: $m / z 449\left(\mathrm{M}^{+}\right)$; Anal. Calcd for $\mathrm{C}_{22} \mathrm{H}_{12} \mathrm{Cl}_{2} \mathrm{~F}_{4} \mathrm{~N}_{2} \mathrm{O}_{3}$ : C, 52.93; H, 2.42; N, 5.61. Found: C, 52.86; H, 2.47; N, 5.70.

(4Z)-4-[(6-Chloro-4-oxo-4H-chromen-3-yl)methylene]5-propyl-2-(2,3,5,6-tetrafluorophenyl)-2,4-dihydro-3Hpyrazol-3-one (5d). Yellow solid; IR $\left(\nu_{\max }, \mathrm{cm}^{-1}\right): 1695$ ( $\mathrm{C}=\mathrm{O}$ lactum), 1655 ( $\mathrm{C}=\mathrm{O}$ chromone), $1605(\mathrm{C}=\mathrm{C}), 1580$ $(\mathrm{C}=\mathrm{N}), 1119(\mathrm{Ar}-\mathrm{F}) ;{ }^{1} \mathrm{H}-\mathrm{NMR} \delta\left(400 \mathrm{MHz}, \mathrm{DMSO}-d_{6}\right)$ : $1.06-1.09(\mathrm{t}, 3 \mathrm{H}, J=12 \mathrm{~Hz}), 1.79-1.85(\mathrm{~m}, 2 \mathrm{H}), 2.71-2.74$ $(\mathrm{t}, 2 \mathrm{H}, J=12 \mathrm{~Hz}), 7.14-7.19(\mathrm{~m}, 1 \mathrm{H}, \mathrm{Ar}-\mathrm{H}), 7.52-7.54(\mathrm{~d}$, $1 \mathrm{H}, J=8 \mathrm{~Hz}, \mathrm{Ar}-\mathrm{H}), 7.69-7.72(\mathrm{~m}, 1 \mathrm{H}, \mathrm{Ar}-\mathrm{H}), 8.08(\mathrm{~s}$, $1 \mathrm{H}, \mathrm{Ar}-\mathrm{H}), 8.25-8.26$ (d, $1 \mathrm{H}, J=4 \mathrm{~Hz}, \mathrm{Ar}-\mathrm{H}), 10.65$ (s, $1 \mathrm{H}$, olefinic proton); MS: $m / z 464\left(\mathrm{M}^{+}\right)$; Anal. Calcd for $\mathrm{C}_{22} \mathrm{H}_{13} \mathrm{ClF}_{4} \mathrm{~N}_{2} \mathrm{O}_{3}$ : C, 56.85; H, 2.82; N, 6.03. Found: C, 56.78; $\mathrm{H}, 2.87$;, 6.11 .

(4Z)-4-[(6-Chloro-7-methyl-4-oxo-4H-chromen-3yl)methylene]-5-propyl-2-(2,3,5,6-tetrafluorophenyl)-2,4dihydro-3H-pyrazol-3-one (5e). Yellow solid; IR ( $\nu_{\max }$, $\left.\mathrm{cm}^{-1}\right)$ : 1711 ( $\mathrm{C}=\mathrm{O}$ lactum), 1649 ( $\mathrm{C}=\mathrm{O}$ chromone), 1630 $(\mathrm{C}=\mathrm{C}), 1588(\mathrm{C}=\mathrm{N}), 1135(\mathrm{Ar}-\mathrm{F}) ;{ }^{1} \mathrm{H}-\mathrm{NMR} \delta(400 \mathrm{MHz}$, DMSO- $\left.d_{6}\right): 1.05-1.09(\mathrm{t}, 3 \mathrm{H}, J=16 \mathrm{~Hz}), 1.79-1.84(\mathrm{~m}, 2 \mathrm{H})$, $2.53\left(\mathrm{~s}, 3 \mathrm{H}, \mathrm{Ar}-\mathrm{CH}_{3}\right), 2.70-2.74(\mathrm{t}, 2 \mathrm{H}, J=16 \mathrm{~Hz}), 7.14-7.18$ (m, 1H, Ar-H), 7.45 (s, 1H, Ar-H), 8.09 (s, 1H, Ar-H), 8.24 (s, $1 \mathrm{H}, \mathrm{Ar}-\mathrm{H}$ ), 10.63 (s, $1 \mathrm{H}$, olefinic proton); MS: $\mathrm{m} / z 478$ $\left(\mathrm{M}^{+}\right)$; Anal. Calcd for $\mathrm{C}_{23} \mathrm{H}_{15} \mathrm{ClF}_{4} \mathrm{~N}_{2} \mathrm{O}_{3}$ : C, 57.69; H, 3.16; N, 5.85. Found: C, 57.60; H, 3.29; N, 5.89.

(4Z)-4-[(4-Oxo-4H-chromen-3-yl)methylene]-2-(2,3,5,6tetrafluorophenyl)-5-(trifluoromethyl)-2,4-dihydro-3H-

pyrazol-3-one $(\mathbf{5 f})$. Yellow solid; IR $\left(v_{\max }, \mathrm{cm}^{-1}\right): 1699(\mathrm{C}=\mathrm{O}$ lactum), $1655(\mathrm{C}=\mathrm{O}$ chromone $), 1625(\mathrm{C}=\mathrm{C}), 1596(\mathrm{C}=\mathrm{N})$, 1133 (Ar-F); ${ }^{1}$ H-NMR $\delta\left(400 \mathrm{MHz}, \mathrm{DMSO}-d_{6}\right): 7.52-7.60$ (m, 3H, Ar-H), 7.77-7.82 (m, 1H, Ar-H), 8.30-8.33 (d, 1H, $J=8 \mathrm{~Hz}, \mathrm{Ar}-\mathrm{H}), 8.53$ (s, 1H, Ar-H), 10.69 (s, 1H, olefinic proton); MS: $m / z 456\left(\mathrm{M}^{+}\right)$; Anal. Calcd for $\mathrm{C}_{20} \mathrm{H}_{7} \mathrm{~F}_{7} \mathrm{~N}_{2} \mathrm{O}_{3}$ : C, 52.65; H, 1.55; N, 6.14. Found: C, 52.53; H, 1.46; N, 6.08.

(4Z)-4-[(6-Methyl-4-oxo-4H-chromen-3-yl)methylene]-2(2,3,5,6-tetrafluorophenyl)-5-(trifluoromethyl)-2,4-dihydro-

3 H-pyrazol-3-one $(5 \mathrm{~g})$. Yellow solid; IR $\left(v_{\max }, \mathrm{cm}^{-1}\right): 1705$ ( $\mathrm{C}=\mathrm{O}$ lactum), 1662 ( $\mathrm{C}=\mathrm{O}$ chromone), $1620(\mathrm{C}=\mathrm{C}), 1593$ $(\mathrm{C}=\mathrm{N}), 1141$ (Ar-F); ${ }^{1} \mathrm{H}-\mathrm{NMR} \delta\left(400 \mathrm{MHz}, \mathrm{DMSO}-d_{6}\right)$ : 2.48 (s, 3H, Ar- $\left.\mathrm{CH}_{3}\right), 7.20-7.23(\mathrm{~m}, 2 \mathrm{H}, \mathrm{Ar}-\mathrm{H}), 7.44-7.46$ (d, $1 \mathrm{H}, J=8 \mathrm{~Hz}, \mathrm{Ar}-\mathrm{H}), 8.06$ (s, $1 \mathrm{H}, \mathrm{Ar}-\mathrm{H}), 8.51$ (s, $1 \mathrm{H}$, Ar-H), 10.64 (s, $1 \mathrm{H}$, olefinic proton); MS: $m / z 470\left(\mathrm{M}^{+}\right)$; Anal. Calcd for $\mathrm{C}_{21} \mathrm{H}_{9} \mathrm{~F}_{7} \mathrm{~N}_{2} \mathrm{O}_{3}$ : C, 53.63; H, 1.93; N, 5.96. Found: C, 53.53; H, 1.89; N, 5.90.

(4Z)-4-[(6,8-Dichloro-4-oxo-4H-chromen-3-yl)methylene]-2(2,3,5,6-tetrafluorophenyl)-5-(trifluoromethyl)-2,4-dihydro-

3 H-pyrazol-3-one $(5 \mathbf{h})$. Yellow solid; IR $\left(\nu_{\max }, \mathrm{cm}^{-1}\right): 1711$ (C=O lactum), 1677 ( $\mathrm{C}=\mathrm{O}$ chromone), 1622 (C=C), 1599 $(\mathrm{C}=\mathrm{N}), 1135$ (Ar-F); ${ }^{1} \mathrm{H}-\mathrm{NMR} \delta\left(400 \mathrm{MHz}, \mathrm{DMSO}-d_{6}\right)$ : 7.52-7.57 (m, 1H, Ar-H), 7.69-7.71 (m, 1H, Ar-H), 8.01 (s, $1 \mathrm{H}, \mathrm{Ar}-\mathrm{H}), 8.40$ (s, 1H, Ar-H), 10.59 (s, 1H, olefinic proton);
MS: $m / z 525\left(\mathrm{M}^{+}\right)$; Anal. Calcd for $\mathrm{C}_{20} \mathrm{H}_{5} \mathrm{Cl}_{2} \mathrm{~F}_{7} \mathrm{~N}_{2} \mathrm{O}_{3}$ : C, 45.74; H, 0.96; N, 5.33. Found: C, 45.61; H, 0.88; N, 5.41.

(4Z)-4-[(6-Chloro-4-oxo-4H-chromen-3-yl)methylene]-2(2,3,5,6-tetrafluorophenyl)-5-(trifluoromethyl)-2,4-dihydro3 H-pyrazol-3-one (5i). Yellow solid;IR $\left(\nu_{\max }, \mathrm{cm}^{-1}\right): 1695$ ( $\mathrm{C}=\mathrm{O}$ lactum), 1671 ( $\mathrm{C}=\mathrm{O}$ chromone), 1611 ( $\mathrm{C}=\mathrm{C}), 1580$ $(\mathrm{C}=\mathrm{N}), 1109(\mathrm{Ar}-\mathrm{F}) ;{ }^{1} \mathrm{H}-\mathrm{NMR} \delta\left(400 \mathrm{MHz}, \mathrm{DMSO}-\mathrm{d}_{6}\right)$ : 7.54-7.56 (m, 2H, Ar-H), 7.72-7.74 (d, 1H, J = 8 Hz, Ar-H), 8.26 (s, 1H, Ar-H), 8.46 (s, 1H, Ar-H), 10.66 (s, 1H, olefinic proton); MS: $m / z 490\left(\mathrm{M}^{+}\right)$; Anal. Calcd for $\mathrm{C}_{20} \mathrm{H}_{6} \mathrm{ClF}_{7} \mathrm{~N}_{2} \mathrm{O}_{3}$ : C, 48.95; H, 1.23; N, 5.71. Found: C, 48.90; H, 1.12; N, 5.64.

2.3. Antimicrobial Activity. The antimicrobial activity of all newly synthesized compounds was evaluated by broth microdilution method according to Clinical and Laboratory Standards Institute (CLSI 2005, formerly known as NCCLS) [48]. The results were determined using minimum inhibitory concentration (MIC) values in $\mu \mathrm{g} / \mathrm{mL}$. Antibacterial activity was screened against two Gram-positive (Staphylococcus aureus MTCC 96 and Streptococcus pyogenes MTCC 443) and two Gram-negative (Escherichia coli MTCC 442 and Pseudomonas aeruginosa MTCC 441) bacterial strains using ampicillin as a standard antibacterial agent. Antifungal activity was screened against three fungal species (Candida albicans MTCC 227, Aspergillus niger MTCC 282, and Aspergillus clavatus MTCC 1323) where griseofulvin was used as a standard antifungal agent. All MTCC cultures were collected from Institute of Microbial Technology, Chandigarh.

Sterile 96-well microtiter plates were used in this assay. All tests were performed in Mueller Hinton broth for bacterial strains and Sabouraud's dextrose broth for fungal strains. Overnight broth cultures of each strain were prepared, and the final inoculum concentration in each well was adjusted to $2 \times 10^{6} \mathrm{CFU} \mathrm{mL}^{-1}$ for the bacterial strains and $2 \times 10^{7}$ $\mathrm{CFU} \mathrm{mL} \mathrm{mL}^{-1}$ for fungal strains. The inoculum concentration was adjusted by diluting broth culture supernatant in sterile distilled water to a density of $0.5 \mathrm{McF}$ arland standards using a nephelometer. Stock solutions of all compounds at $2000 \mu \mathrm{g} / \mathrm{mL}$ were prepared by dissolving them in DMSO. Serial dilutions were prepared in primary and secondary screening. In primary screening, 500, 250, and $200 \mu \mathrm{g} / \mathrm{mL}$ concentrations of the synthesized compounds were taken. The compounds found active in primary screening were further tested in a second set of dilutions against all microorganisms. The compounds found active in primary screening were similarly diluted to obtain $100,62.5,50$, and $25 \mu \mathrm{g} / \mathrm{mL}$ concentrations. In the tests, triphenyltetrazolium chloride (TTC) was also added to culture medium as growth indicator. The final concentration of TTC after inoculation was $0.05 \%$. The microbial growth was determined after $24 \mathrm{~h}$ incubation at $37^{\circ} \mathrm{C}$ for the bacteria and at $25^{\circ} \mathrm{C}$ after $48 \mathrm{~h}$ for the fungi. The MIC is defined as the lowest concentration of a compound at which the microorganism does not demonstrate visible growth. All determinations were performed in duplicate to check the accuracy of results. The antimicrobial activity results (MIC) are presented in Table 3. 
TABLE 3: Antibacterial and antifungal data of newly synthesized pyrazole-pyrazolone (4a-j) and chromone-pyrazolone (5a-i) indicated by MIC values $(\mu \mathrm{g} / \mathrm{mL})$.

\begin{tabular}{|c|c|c|c|c|c|c|c|}
\hline \multirow{3}{*}{ Compound } & \multicolumn{7}{|c|}{ Minimum inhibitory concentration (MIC, $\mu \mathrm{g} / \mathrm{mL}$ ) } \\
\hline & \multicolumn{2}{|c|}{ Gram-positive bacteria } & \multicolumn{2}{|c|}{ Gram-negative bacteria } & \multicolumn{3}{|c|}{ Fungi } \\
\hline & S. aureus & S. pyogenes & E. coli & P. aeruginosa & C. albicans & A. niger & A. clavatus \\
\hline $4 \mathbf{a}$ & 250 & 200 & 200 & 100 & $>1000$ & $>1000$ & $>1000$ \\
\hline $4 b$ & 125 & 100 & 62.5 & 100 & 500 & 1000 & 1000 \\
\hline $4 c$ & 200 & 250 & 100 & 125 & 1000 & 1000 & 1000 \\
\hline $4 d$ & 100 & 100 & 62.5 & 200 & 1000 & $>1000$ & $>1000$ \\
\hline $4 e$ & 125 & 62.5 & 200 & 250 & $>1000$ & $>1000$ & $>1000$ \\
\hline $4 \mathrm{f}$ & 200 & 250 & 250 & 250 & 1000 & 500 & 500 \\
\hline $4 g$ & 250 & 200 & 100 & 200 & $>1000$ & 250 & 500 \\
\hline $4 h$ & 500 & 250 & 250 & 125 & $>1000$ & 500 & 500 \\
\hline $4 \mathbf{i}$ & 500 & 500 & 200 & 200 & 500 & $>1000$ & $>1000$ \\
\hline $4 j$ & 250 & 500 & 125 & 250 & 500 & 1000 & 1000 \\
\hline $5 \mathbf{a}$ & 200 & 250 & 250 & 125 & 500 & 1000 & 1000 \\
\hline $5 b$ & 100 & 62.5 & 200 & 100 & 1000 & $>1000$ & $>1000$ \\
\hline $5 c$ & 125 & 100 & 250 & 200 & 1000 & $>1000$ & $>1000$ \\
\hline $5 d$ & 200 & 200 & 250 & 200 & $>1000$ & 500 & 500 \\
\hline $5 e$ & 100 & 100 & 500 & 200 & 250 & 1000 & $>1000$ \\
\hline $5 f$ & 62.5 & 200 & 500 & 500 & 250 & $>1000$ & $>1000$ \\
\hline $5 \mathrm{~g}$ & 100 & 250 & 200 & 200 & 500 & $>1000$ & $>1000$ \\
\hline $5 \mathrm{~h}$ & 250 & 200 & 100 & 250 & 1000 & $>1000$ & $>1000$ \\
\hline $5 \mathbf{i}$ & 250 & 200 & 125 & 62.5 & 1000 & 500 & 1000 \\
\hline Ampicillin & 250 & 100 & 100 & 100 & - & - & - \\
\hline Griseofulvin & - & - & - & - & 500 & 100 & 100 \\
\hline
\end{tabular}

\section{Result and Discussion}

3.1. Chemistry. In the present investigation, our aim was to do the synthesis of multifluorinated pyrazole-pyrazolone (4) and chromone-pyrazolone (5) by using green technique such as ultrasonication and investigate their antibacterial and antifungal activities (Scheme 1). The starting material multifluorinated pyrazolone (3) was prepared by literature known procedure [49] using $\beta$-ketoester (1) and 2,3,5,6-tetra-fluoro phenyl hydrazine (2). The pyrazolone (3) was treated with 3formyl chromone or 4-formyl pyrazole in acetic acid under conventional heating as well as ultrasound irradiation to give pyrazole-pyrazolone (4) and chromone-pyrazolone (5). The structures of all compounds were confirmed on the basis of spectral analysis. The IR spectra of compounds 4 and 5 showed a peak in the range $1680-1711 \mathrm{~cm}^{-1}$ for $\mathrm{C}=\mathrm{O}$ stretching of five membered lactum ring. They also exhibited a peak in the range $1500-1600 \mathrm{~cm}^{-1}$ for $\mathrm{C}=\mathrm{N}$ stretching. The ${ }^{1} \mathrm{H}-\mathrm{NMR}$ spectra of compounds $\mathbf{4 a - e}$ and $\mathbf{5 a - e}$ clearly showed peaks for $\mathrm{CH}_{3}-\mathrm{CH}_{2}-\mathrm{CH}_{2}$-(propyl group) protons, while such peaks were absent in compounds $\mathbf{4 f}-\mathbf{j}$ and $\mathbf{5 f}-\mathbf{i}$ as they have no propyl group. The exocyclic methylene group proton appeared as a singlet in the range of 10.11-10.66 $\delta$. The aromatic protons present in all compounds were also confirmed by ${ }^{1} \mathrm{H}-\mathrm{NMR}$ spectra. Further the structures of all compounds were also confirmed by molecular ion peak in their mass spectra. For all the synthesized compounds, elemental analysis values are in good agreement with theoretical data.

It was observed that ultrasound irradiation method was practically more superior than conventional heating method in terms of higher yields, rapid, and environmentally benign process which makes this protocol more useful.

3.2. Antimicrobial Activity. Antibacterial activity was screened against Staphylococcus aureus, Streptococcus pyogenes, Escherichia coli, and Pseudomonas aeruginosa bacterial strains using ampicillin as a standard antibacterial agent. Antifungal activity was screened against fungal species Candida albicans, Aspergillus niger, and Aspergillus clavatus where griseofulvin was used as a standard antifungal agent.

Reviewing the antibacterial activity data (Table 1) of all newly synthesized pyrazole-pyrazolone $(\mathbf{4} \mathbf{a}-\mathbf{j})$ and chromone-pyrazolone (5a-i), it was revealed that the compounds (4b, 4d), (5i), (5f), and $(4 \mathbf{e}, 5 \mathbf{b})$ showed highest activity, that is, $62.5 \mu \mathrm{g} / \mathrm{mL}$ against E. coli, P. aeruginosa, $S$. aureus, and $S$. pyogenes, respectively. Among pyrazolepyrazolone, the compounds $\mathbf{4 b}, \mathbf{4 c}, \mathbf{4 d}, \mathbf{4 e}$ containing propyl group and compound $\mathbf{4 f}$ containing trifluoromethyl group, similarly among chromone-pyrazolone, the compounds $\mathbf{5 a}$, $\mathbf{5 b}, \mathbf{5 c}, \mathbf{5 d}, \mathbf{5 e}$ containing propyl group and compounds $\mathbf{5 f}$, $5 \mathrm{~g}$ containing trifluoromethyl group are found to possess 
high potency against Gram-Positive S. aureus bacterial strain as compared with standard bactericidal ampicillin. So it is concluded that compounds with propyl group rather than $\mathrm{CF}_{3}$ group exhibited high potency against gram positive $S$. aureus bacterial strain. It is also observed that compounds (4c, 4g, 5h), (4a, 4b, 5b), (4a, 4g, 4j, 5h, 5i), and (4b, 4d, 5c, 5e) showed promising activity against E. coli, P. aeruginosa, $S$. aureus, and S. pyogenes, respectively, as compared with standard ampicillin drug.

Antifungal activity study of synthesized compounds reveals that compounds $\mathbf{5 e}$ and $\mathbf{5} \mathbf{f}$ exhibited more potency while compounds $\mathbf{4 b}, \mathbf{4 i}, \mathbf{4 j}, 5 \mathbf{a}$, and $5 \mathbf{g}$ showed good antifungal activity against $C$. albicans as compared with standard fungicidal griseofulvin. All the synthesized compounds showed moderate-to-poor antifungal activity profile against A. niger and A. clavatus fungal strains.

\section{Conclusion}

Present study describes synthesis of a series of novel multifluorinated pyrazolone containing pyrazole and chromone nucleus by conventional heating as well as ultrasonication technique. The ultrasonication method was more efficient in terms of short reaction time, high yields, and clean reaction process. The antimicrobial activity result reveals that some of the synthesized compounds exhibited promising activity against the tested bacterial and fungal strains. The insights gained in this study will be useful for further studies on potential anti-infective agents. A study regarding structure activity relationship is in progress.

\section{Acknowledgments}

The authors are thankful to Dr. S. R. Walunj (Principal, PVP, College), Dr. T. N. Gholap (Principal, RKMM, Ahmednagar), and Management, PRES, for providing necessary facilities and constant encouragement. The authors also thank SAIF, Chandigarh, for spectral analysis.

\section{References}

[1] N. Woodford, "Novel agents for the treatment of resistant Gram-positive infections," Expert Opinion on Investigational Drugs, vol. 12, no. 2, pp. 117-137, 2003.

[2] L. Kuznetsova, M. I. Ungureau, and A. Pepe, "Trifluoromethyland difluoromethyl- $\beta$-lactams as useful building blocks for the synthesis of fluorinated amino acids, dipeptides, and fluorotaxoids," Journal of Fluorine Chemistry, vol. 125, no. 4, pp. 415-500, 2004.

[3] M. A. Al-Haiza, S. A. El-Assiery, and G. H. Sayed, "Synthesis and potential antimicrobial activity of some new compounds containing the pyrazol-3-one moiety," Acta Pharmaceutica, vol. 51, no. 4, pp. 251-261, 2001.

[4] F. Moreau, N. Desroy, J. M. Genevard et al., "Discovery of new Gram-negativeantivirulence drugs: structure and properties of novel E. coli WaaC inhibitors," Bioorganic and Medicinal Chemistry Letters, vol. 18, no. 14, pp. 4022-4026, 2008.
[5] R. N. Mahajan, F. H. Havaldar, and P. S. Fernandes, "Syntheses and biological activity of heterocycles derived from 3methoxy-1-phenyl-1H-pyrazole-5-carboxylate," Journal of the Indian Chemical Society, vol. 68, no. 4, pp. 245-246, 1991.

[6] D. Castagnolo, F. Manetti, M. Radi et al., "Synthesis, biological evaluation, and SAR study of novel pyrazole analogues as inhibitors of Mycobacterium tuberculosis: part 2. Synthesis of rigid pyrazolones," Bioorganic and Medicinal Chemistry, vol. 17, no. 15, pp. 5716-5721, 2009.

[7] M. Radi, V. Bernardo, B. Bechi, D. Castagnolo, M. Pagano, and M. Botta, "Microwave-assisted organocatalytic multicomponent Knoevenagel/hetero Diels-Alder reaction for the synthesis of 2,3-dihydropyran[2,3-c]pyrazoles," Tetrahedron Letters, vol. 50, no. 47, pp. 6572-6575, 2009.

[8] E. A. M. Badawey and I. M. El-Ashmawey, "Nonsteroidal antiinflammatory agents-part 1: antiinflammatory, analgesic and antipyretic activity of some new 1-(pyrimidin2-yl)-3-pyrazolin-5-ones and 2-(pyrimidin-2-yl)-1,2,4,5,6,7hexahydro-3H-indazol-3-ones," European Journal of Medicinal Chemistry, vol. 33, no. 5, pp. 349-361, 1998.

[9] A. Tantawy, H. Eisa, A. Ismail, and M. E. Alexandria, "Synthesis of 1-(substituted) 4-arylhydrazono-3-methyl-2-pyrazolin-5ones as potential antiinflammatory agents," Journal of Pharmaceutical Sciences, vol. 2, p. 133, 1988.

[10] F. A. Pasha, M. Muddassar, M. M. Neaz, and S. J. Cho, "Pharmacophore and docking-based combined in-silico study of KDR inhibitors," Journal of Molecular Graphics and Modelling, vol. 28, no. 1, pp. 54-61, 2009.

[11] C. E. Rosiere and M. I. Grossman, "An analog of histamine that stimulates gastric acid secretion without other actions of histamine," Science, vol. 113, no. 2945, p. 651, 1951.

[12] D. M. Bailey, P. E. Hansen, A. G. Hlavac et al., "3,4Diphenyl-1H-pyrazole-1-propanamine antidepressants," Journal of Medicinal Chemistry, vol. 28, no. 2, pp. 256-260, 1985.

[13] P. M. S. Chauhan, S. Singh, and R. K. Chatterjee, "Antifilarial profile of substituted pyrazoles: a new class of antifilarial agents," Indian Journal of Chemistry B, vol. 32, pp. 858-861, 1993.

[14] X. Zhang, Y. Kluger, Y. Nakayama et al., "Gene expression in mature neutrophils: early responses to inflammatory stimuli," Journal of Leukocyte Biology, vol. 75, no. 2, pp. 358-372, 2004.

[15] M. T. Quinn and K. A. Gauss, "Structure and regulation of the neutrophil respiratory burst oxidase: comparison with nonphagocyte oxidases," Journal of Leukocyte Biology, vol. 76, pp. 760-781, 2004.

[16] S. J. Weiss, “Tissue destruction by neutrophils," The New England Journal of Medicine, vol. 320, pp. 365-376, 1989.

[17] M. Himly, B. Jahn-Schmid, K. Pittertschatscher et al., "Ig E-mediated immediate-type hypersensitivity to the pyrazolone drug propyphenazone," Journal of Allergy and Clinical Immunology, vol. 111, no. 4, pp. 882-888, 2003.

[18] T. Watanabe, S. Yuki, M. Egawa, and H. Nishi, "Protective effects of MCI-186 on cerebral ischemia: possible involvement of free radical scavenging and antioxidant actions," Journal of Pharmacology and Experimental Therapeutics, vol. 268, pp. 1597-1604, 1994

[19] H. Kawai, H. Nakai, M. Suga, S. Yuki, T. Watanabe, and K. I. Saito, "Effects of a novel free radical scavenger, MCI-186, on ischemic brain damage in the rat distal middle cerebral artery occlusion model," Journal of Pharmacology and Experimental Therapeutics, vol. 281, no. 2, pp. 921-927, 1997. 
[20] T. W. Wu, L. H. Zeng, J. Wu, and K. P. Fung, "Myocardial protection of MCI-186 in rabbit ischemia-reperfusion," Life Sciences, vol. 71, no. 19, pp. 2249-2255, 2002.

[21] R. Aggarwal, V. Kumar, P. Tyagi, and S. P. Singh, "Synthesis and antibacterial activity of some new 1-heteroaryl-5-amino-3H/ methyl-4-phenylpyrazoles," Bioorganic and Medicinal Chemistry, vol. 14, no. 6, pp. 1785-1791, 2006.

[22] V. Kumar, R. Aggarwal, P. Tyagi, and S. P. Singh, "Synthesis and antibacterial activity of some new 1-heteroaryl-5-amino-4phenyl-3-trifluoromethylpyrazoles," European Journal of Medicinal Chemistry, vol. 40, no. 9, pp. 922-927, 2005.

[23] J. L. Kane, B. H. Hirth, B. Liang, B. B. Gourlie, S. Nahill, and G. Barsomian, "Ureas of 5-aminopyrazole and 2-aminothiazole inhibit growth of gram-positive bacteria," Bioorganic and Medicinal Chemistry Letters, vol. 13, no. 24, pp. 4463-4466, 2003.

[24] P. P. Deohate, J. P. Deohate, and B. N. Berad, "Synthesis of some novel 1,2,4-dithiazolidines and their antibacterial and antifungal activity," Asian Journal of Chemistry, vol. 16, no. 1, pp. 255-260, 2004.

[25] O. Prakash, R. Kumar, and V. Prakash, "Synthesis and antifungal activity of some new 3-hydroxy-2-(1-phenyl-3-aryl-4pyrazolyl) chromones," European Journal of Medicinal Chemistry, vol. 43, pp. 435-440, 2008.

[26] K. L. Kees, J. J. Fitzgerald, K. E. Steiner et al., "New potent antihyperglycemic agents in $\mathrm{db} / \mathrm{db}$ mice: synthesis and structure-activity relationship studies of (4-substituted benzyl)(trifluoromethyl)pyrazoles and -pyrazolones," Journal of Medicinal Chemistry, vol. 39, no. 20, pp. 3920-3928, 1996.

[27] G. Meazza, F. Bettarini, P. La Porta et al., "Synthesis and herbicidal activity of novel heterocyclic protoporphyrinogen oxidase inhibitors," Pest Management Science, vol. 60, no. 12, pp. 1178-1188, 2004.

[28] T. W. Waldrep, J. R. Beck, M. P. Lynch, and F. L. Wright, "Synthesis and herbicidal activity of 1-aryl-5-halo- and 1-aryl5-(trifluoromethyl)-1H-pyrazole-4-carboxamides," Journal of Agricultural and Food Chemistry, vol. 38, no. 2, pp. 541-544, 1990.

[29] D. J. Wustrow, T. Capiris, R. Rubin et al., "Pyrazolo[1,5a]pyrimidine CRF-1 receptor antagonists," Bioorganic and Medicinal Chemistry Letters, vol. 8, no. 16, pp. 2067-2070, 1998.

[30] A. G. Habeeb, P. N. Praveen Rao, and E. E. Knaus, "Design and synthesis of celecoxib and rofecoxib analogues as selective cyclooxygenase-2 (COX-2) inhibitors: replacement of sulfonamide and methylsulfonyl pharmacophores by an azido bioisostere," Journal of Medicinal Chemistry, vol. 44, no. 18, pp. 3039-3042, 2001.

[31] A. M. Martel, A. Graul, X. Rabasseda, and R. Castañer, "Sildenafil," Drugs of the Future, vol. 22, no. 2, pp. 138-143, 1997.

[32] A. S. Bhat, J. L. Whetstone, and R. W. Brueggemeier, "Novel synthetic routes suitable for constructing benzopyrone combinatorial libraries," Tetrahedron Letters, vol. 40, no. 13, pp. 2469-2472, 1999.

[33] B. Bauvois, M. L. Puiffe, J. B. Bongui, S. Paillat, C. Monneret, and D. Dauzonne, "Synthesis and biological evaluation of novel flavone-8-acetic acid derivatives as reversible inhibitors of aminopeptidase N/CD13," Journal of Medicinal Chemistry, vol. 46, no. 18, pp. 3900-3913, 2003.

[34] H. P. Kim, K. H. Son, H. W. Chang, and S. S. Kang, "Antiinflammatory plant flavonoids and cellular action mechanisms," Journal of Pharmacological Sciences, vol. 96, no. 3, pp. 229-245, 2004.
[35] E. Middleton, C. Kandaswami, and T. C. Theoharides, "The effects of plant flavonoids on mammalian cells: implications for inflammation, heart disease, and cancer," Pharmacological Reviews, vol. 52, no. 4, pp. 673-751, 2000.

[36] C. J. Bennett, S. T. Caldwell, D. B. McPhail, P. C. Morrice, G. G. Duthie, and R. C. Hartley, "Potential therapeutic antioxidants that combine the radical scavenging ability of myricetin and the lipophilic chain of vitamin E to effectively inhibit microsomal lipid peroxidation," Bioorganic and Medicinal Chemistry, vol. 12, no. 9, pp. 2079-2098, 2004.

[37] V. Krishnamachari, L. H. Levine, C. Zhou, and P. W. Pare, "In vitro flavon-3-ol oxidation mediated by a $\mathrm{B}$ ring hydroxylation pattern," Chemical Research in Toxicology, vol. 17, no. 6, pp. 795-804, 2004.

[38] E. Knoevenagel, "Condensation von Malonsure mit aromatischen Aldehyden durch Ammoniak und Amine," Berichte der deutschen chemischen Gesellschaft, vol. 31, no. 3, pp. 2596-2619, 1898.

[39] E. Castelli, G. Cascio, and E. Manghisi, WO, 9807698, 1988.

[40] A. J. Kesel and W. Oberthur, WO, 9820013, 1998.

[41] A. Gaplovsky, M. Gaplovsky, S. Toma, and J. L. Luche, "Ultrasound effects on the photopinacolization of benzophenone," Journal of Organic Chemistry, vol. 65, no. 25, pp. 8444-8447, 2000.

[42] R. Rajgopal, D. V. Jarikote, and K. V. Srinivasan, "Ultrasound prompted Suzuki cross-coupling reactions in ionic liquids at ambient conditions," Chemical Communications, no. 6, pp. 616-617, 2002.

[43] B. A. Song, G. P. Zhang, S. Yang, D. Y. Hu, and L. H. Jin, "Synthesis of N-(4-bromo-2-trifluoromethylphenyl)-1-(2fluorophenyl)-O,O-dialkyl- $\alpha$-aminophosphonates under ultrasonic irradiation," UltraChem, vol. 13, p. 1544, 2001.

[44] S. S. Shindalkar, B. R. Madje, and M. S. Shingare, "A simple procedure for the preparation of acylals from 4-oxo- $(4 \mathrm{H})-1$ benzopyran-3-carboxaldehyde using envirocat EPZ10R catalyst under ultrasonic irradiation," Indian Journal of Heterocyclic Chemistry, vol. 15, no. 1, pp. 81-82, 2005.

[45] B. K. Karale and A. G. Gadhave, "Synthesis and antibacterial activity of some spiroisoxazolines," Indian Journal of Heterocyclic Chemistry, vol. 19, no. 4, pp. 389-392, 2010.

[46] R. B. Gaikar, A. G. Gadhave, and B. K. Karale, "Synthesis of some biologically active pyrazolones," Indian Journal of Heterocyclic Chemistry, vol. 19, no. 4, pp. 325-328, 2010.

[47] A. V. Gadakh, C. Pandit, S. S. Rindhe, and B. K. Karale, "Synthesis and antimicrobial activity of novel fluorine containing 4-(substituted-2-hydroxybenzoyl)-1H-pyrazoles and pyrazolyl benzo[d]oxazoles," Bioorganic and Medicinal Chemistry Letters, vol. 20, no. 18, pp. 5572-5576, 2010.

[48] Clinical and Laboratory Standards Institute (CLSI), "Performance standards for antimicrobial susceptibility testing: 15th informational supplement," CLSI Document M100-S15, Wayne, Pa, USA, 2005.

[49] B. S. Furniss, A. J. Hannaford, P. W. G. Smith, and A. R. Patchel, Vogels Text Book of Practical Organic Chemistry, Pearson Education, 5th edition, 2007. 

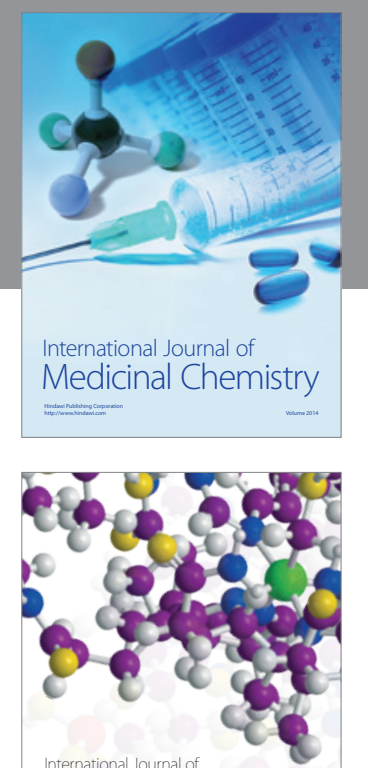

\section{Carbohydrate} Chemistry

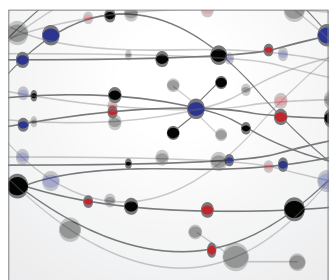

The Scientific World Journal
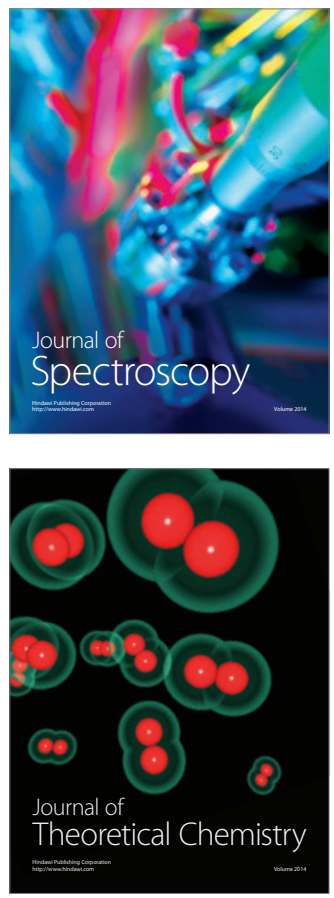
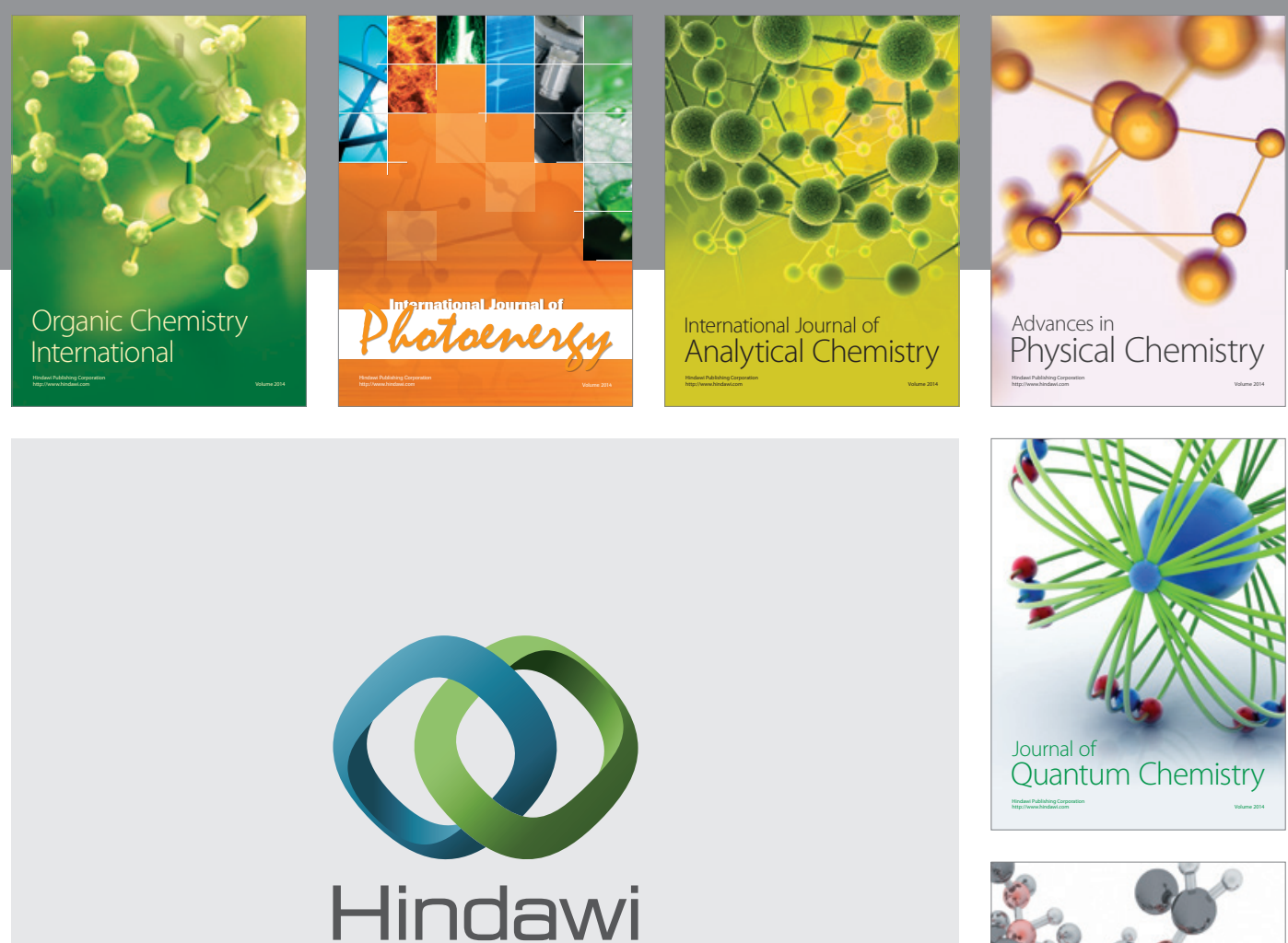

Submit your manuscripts at

http://www.hindawi.com

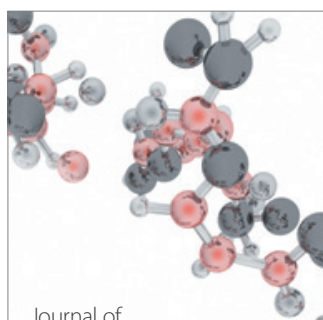

Analytical Methods

in Chemistry

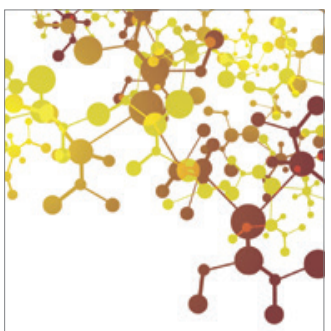

Journal of

Applied Chemistry

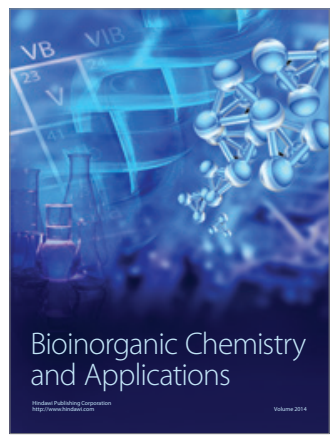

Inorganic Chemistry
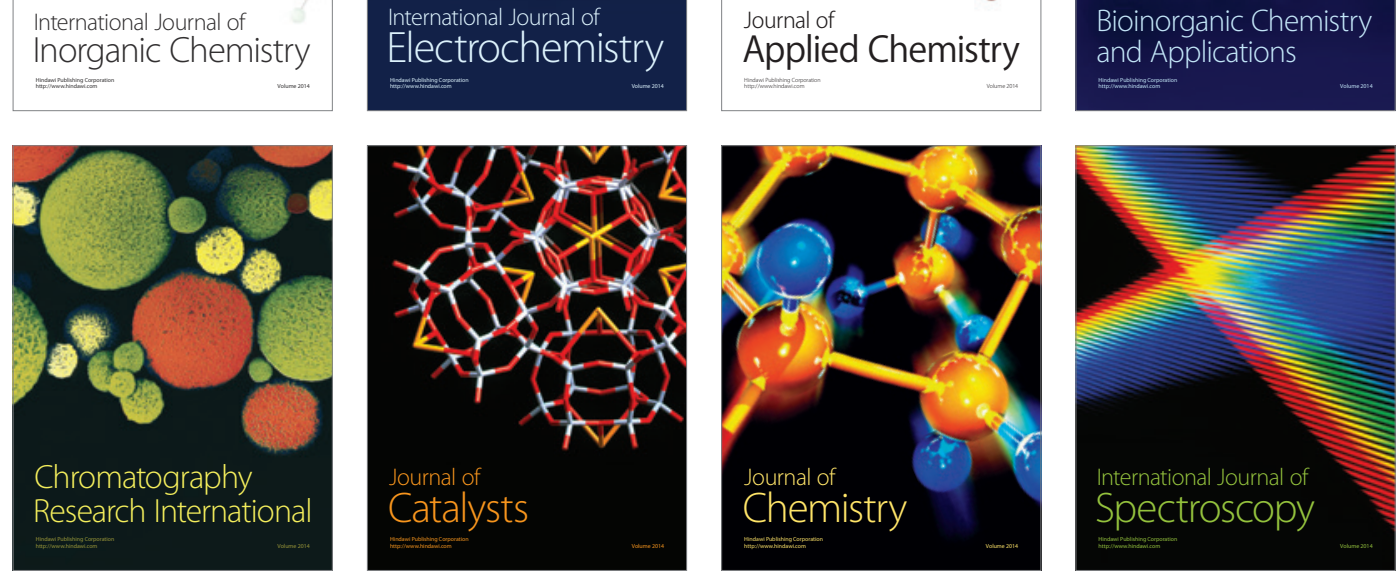